\title{
Alkali Metal Intercalation in MXene/Graphene Heterostructures: A New Platform for Ion Battery Applications
}

\author{
Ilker Demiroglu, ${ }^{\dagger}$ François M. Peeters, ${ }^{\ddagger}$ Ŏguz Gülseren, ${ }^{\S}$ Deniz Çakır, ${ }^{\#(0)}$ and Cem Sevik*, ${ }^{\dagger}$ \\ ${ }^{\dagger}$ Department of Mechanical Engineering, Faculty of Engineering, Eskişehir Technical University, Eskisehir, TR 26555, Turkey \\ ${ }^{*}$ Department of Physics, University of Antwerp, Groenenborgerlaan 171, 2020 Antwerpen, Belgium \\ ${ }^{\S}$ Department of Physics, Bilkent University, Ankara 06800, Bilkent, Turkey \\ \#Department of Physics and Astrophysics, University of North Dakota, Grand Forks, North Dakota 58202, United States
}

\section{Supporting Information}

\begin{abstract}
The adsorption and diffusion of $\mathrm{Na}, \mathrm{K}$, and $\mathrm{Ca}$ atoms on MXene/ graphene heterostructures of MXene systems $\mathrm{Sc}_{2} \mathrm{C}(\mathrm{OH})_{2}, \mathrm{Ti}_{2} \mathrm{CO}_{2}$, and $\mathrm{V}_{2} \mathrm{CO}_{2}$ are systematically investigated by using first-principles methods. We found that alkali metal intercalation is energetically favorable and thermally stable for $\mathrm{Ti}_{2} \mathrm{CO}_{2}$ / graphene and $\mathrm{V}_{2} \mathrm{CO}_{2}$ /graphene heterostructures but not for $\mathrm{Sc}_{2} \mathrm{C}(\mathrm{OH})_{2}$. Diffusion kinetics calculations showed the advantage of $\mathrm{MXene/graphene} \mathrm{heterostructures} \mathrm{over}$ sole MXene systems as the energy barriers are halved for the considered alkali metals. Low energy barriers are found for $\mathrm{Na}$ and $\mathrm{K}$ ions, which are promising for fast charge/ discharge rates. Calculated voltage profiles reveal that estimated high capacities can be fully achieved for $\mathrm{Na}$ ion in $\mathrm{V}_{2} \mathrm{CO}_{2}$ /graphene and $\mathrm{Ti}_{2} \mathrm{CO}_{2}$ /graphene heterostructures. Our results indicate that $\mathrm{Ti}_{2} \mathrm{CO}_{2}$ /graphene and $\mathrm{V}_{2} \mathrm{CO}_{2}$ /graphene electrode materials are very promising for $\mathrm{Na}$ ion battery applications. The former could be exploited for low voltage applications while the latter will be more appropriate for higher voltages.
\end{abstract}

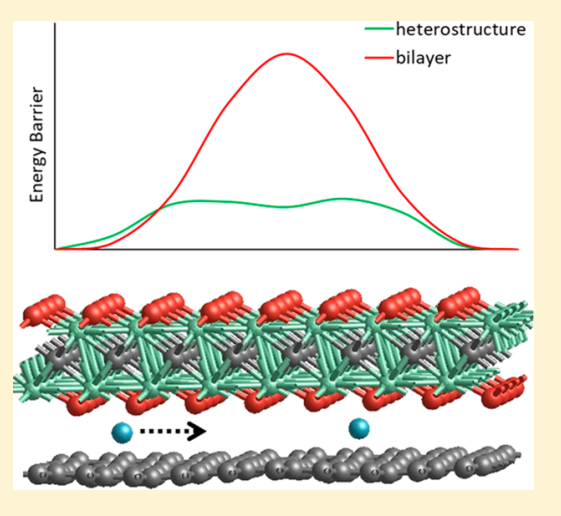

T $\mathrm{n}$ the past decade, Li-ion batteries (LIBs) have been widely 1 used for electrochemical energy storage in portable electronic devices and electric vehicles (EVs). However, the rarity of $\mathrm{Li}$ resources are expected to prevent the extensive usage of large scale EVs powered by LIBs due to high cost. ${ }^{1}$ Therefore, it is critical to develop battery technologies based on alternative earth abundant elements. Such candidates may be abundant metal elements positioned around the Li element in the periodic table, which share similar alkali metal chemistry. One such example is the development of $\mathrm{Na}$ ion batteries (NIBs) for which significant progress has been already made by using the knowledge on LIBs. ${ }^{2-4}$

Still to date, the energy density and rate capability of LIBs and NIBs are insufficient to satisfy customers' needs and the demand for metal based new generation batteries that have good cycling stability, long life span, and large reversible energy/power capacity is growing. Thus, rechargeable batteries based on $2 \mathrm{D}$ materials have started to receive great attention as promising anode materials since their enhanced gravimetric and volumetric capacities due to high surface area and capability of higher charging rates as metal ions do not need to diffuse in a $3 \mathrm{D}$ lattice. For example, $\mathrm{Mo}_{2} \mathrm{C}$ was shown to exhibit superior electrochemical properties in lithium-ion battery applications. ${ }^{5}$ Other $2 \mathrm{D}$ layered materials have been also widely investigated and high energy storage density and high rate capacity were theoretically predicted for transition metal dichalcogenides, ${ }^{6,7}$ black phosphorus, ${ }^{8,9}$ and MXenes. ${ }^{10-17}$
Up to now most of the theoretical and experimental works have been focused on single $2 \mathrm{D}$ materials, which has the disadvantage of fading device performance over time due to restacking during the cycling process. Heterostructures of $2 \mathrm{D}$ materials are an alternative to remedy this shortcoming with an increased the device performance that benefits from a synergy between different materials. ${ }^{18-20}$ For example, a lithium-ion capacity in excess of $750 \mathrm{mAh} / \mathrm{g}$ has been demonstrated for batteries with MXene electrodes optimized with CNTs. ${ }^{21}$ Furthermore, heterostructures with adjustable interlayer distance may accommodate much larger (i.e., $\mathrm{Na}^{+}, \mathrm{K}^{+}$) and multivalent ions (i.e., $\mathrm{Ca}^{2+}$ ) and therefore are a promising alternative for the replacement of the $\mathrm{Li}$ in current ion batteries. In addition, stacking different materials together can be a promising way to increase the conductivity of individual layers, which was recently experimentally confirmed for graphene/MXene heterostructures. $^{22}$

Recent advances in assembly techniques for 2D materials made fabrication of MXene/graphene heterostructures possible with high electrochemical performance. ${ }^{23-28}$ However, despite previous theoretical studies, ${ }^{18,29,30}$ more insight into the MXene/graphene interface and its interaction with the alkali metal ions is needed for the rational design of superior devices.

Received: October 4, 2018

Accepted: January 29, 2019

Published: January 29, 2019 


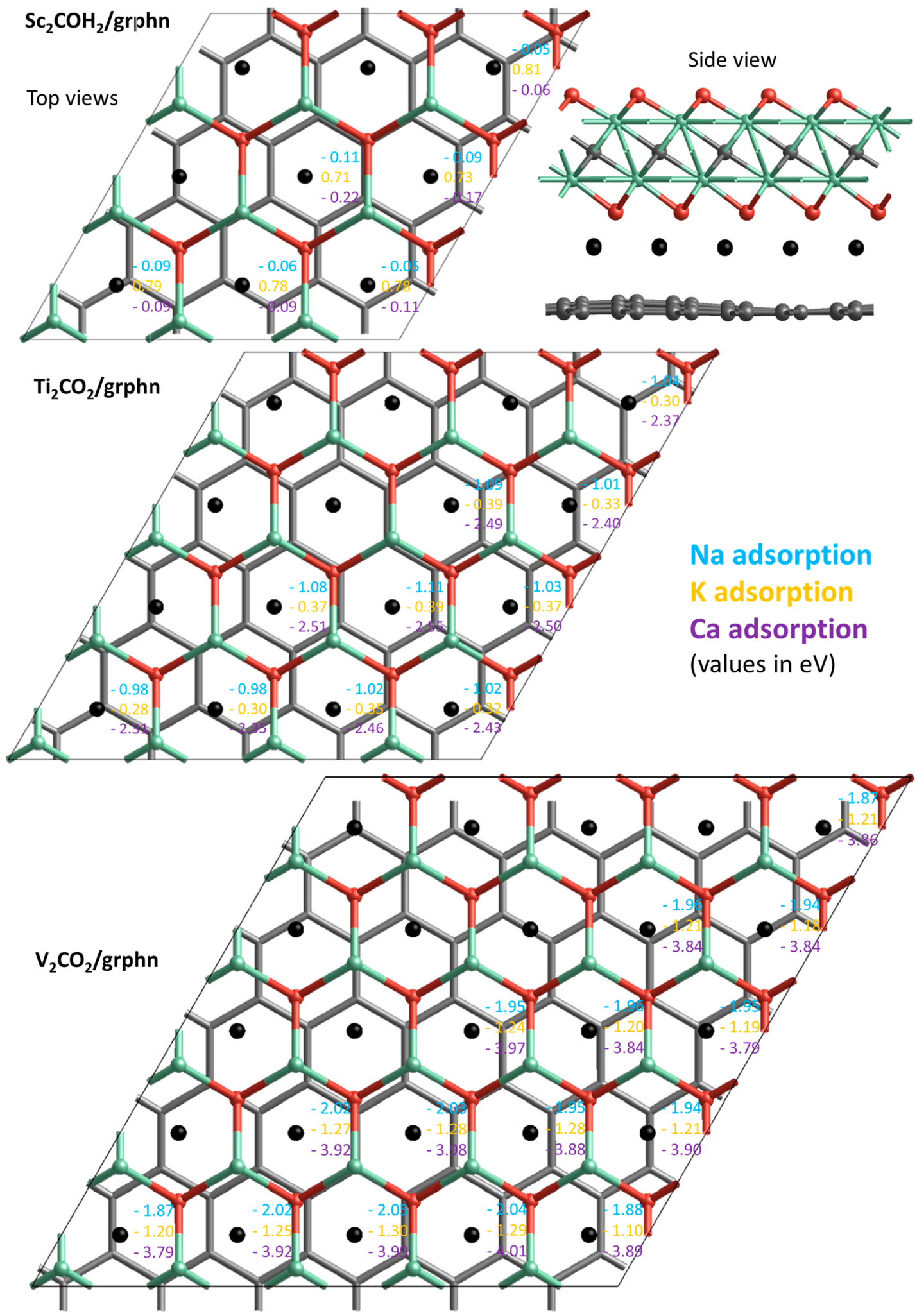

Figure 1. Locations (black spheres) of alkali atoms intercalated in MXene/graphene heterostructures and their corresponding binding energy values. Green, gray, and red spheres stand for metal $(\mathrm{Sc}, \mathrm{Ti}$, or $\mathrm{V})$, carbon, and oxygen atoms, respectively.

In this study, by combining superior properties of MXenes with outstanding electrical conductivity of graphene, we explored its potential in battery applications alternative to LIBs. Following our previous study, ${ }^{18}$ we considered $\mathrm{OH}$ terminated $\mathrm{Sc}_{2} \mathrm{C}$ and $\mathrm{O}$ terminated $\mathrm{Ti}_{2} \mathrm{C}$ and $\mathrm{V}_{2} \mathrm{C}$ MXene systems and we systematically investigated the intercalation of alkali metals $(\mathrm{Na}, \mathrm{K}$, and $\mathrm{Ca}$ ) in van der Waals heterostructures of MXenes and graphene by $a b$ initio methods. O terminated systems are primarily selected due to their superior performance over other termination groups such as $\mathrm{F}$ and $\mathrm{OH}_{;}{ }^{29}$ however, $\mathrm{O}$ termination is found not to be stable for the $\mathrm{Sc}_{2} \mathrm{C}$ system. $^{18}$ 
All the total energy calculations are performed by using density functional theory (DFT) within the Vienna ab initio simulation package (VASP). ${ }^{31,32}$ The generalized gradient approximation (GGA) is used in the Perdew-BurkeErnzerhof $(\mathrm{PBE})^{33}$ formulation scheme. The projector augmented wave (PAW $)^{34,35}$ method is used to describe the core states and the valence states are represented by a plane wave basis with a $500 \mathrm{eV}$ cutoff. Heterostructures were separated by over $15 \AA$ in the stacking direction to avoid spurious periodic interactions while the van der Waals correction of Grimme (D3) ${ }^{36}$ with Becke-Jonson damping ${ }^{37}$ is adopted to model the interaction at the interface. The Gamma-centered $3 \times 3 \times 1$ reciprocal space $k$-point sampling is used for all supercells. The convergence criterion for electronic and ionic relaxations are set as $10^{-6} \mathrm{eV}$ and $10^{-2} \mathrm{eV} /$ $\AA$, respectively. Diffusion energy barriers are calculated by climbing nudged elastic band ( $\mathrm{cNEB})^{38,39}$ method by using a seven-image pathway. The isothermal-isobaric $(N P T)^{40,41}$ ensemble has been selected for $a b$ initio molecular dynamics $^{42,43}$ (MD) simulations. We only applied the constant pressure algorithm to the two lattice vectors parallel to the $2 \mathrm{D}$ plane, leaving the third vector unchanged during the simulation. External pressure is kept at $0 \mathrm{~Pa}$. Each $a b$ initio MD simulation lasted 17 ps with a time step of $1 \mathrm{fs}$, and the temperature is kept constant at $300 \mathrm{~K}$. The friction coefficients of atomic and lattice degrees of freedom is set to $5 \mathrm{ps}^{-1}$.

MXene structures $\left(\mathrm{M}_{n+1} \mathrm{C}_{n} \mathrm{~T}_{x}\right)$ consist of a metal carbide $\left(\mathrm{M}_{n+1} \mathrm{C}_{n}\right)$ core together with termination groups $\left(\mathrm{T}_{x}\right)$ such as $\mathrm{O}$ and $\mathrm{OH}$ in a hexagonal lattice. To minimize the lattice mismatch with graphene, $3 \times 3$ supercell of $\mathrm{Sc}_{2} \mathrm{C}(\mathrm{OH})_{2}$ with a $4 \times 4$ supercell of graphene, a $4 \times 4$ supercell of $\mathrm{Ti}_{2} \mathrm{CO}_{2}$ or $\mathrm{Ti}_{2} \mathrm{C}(\mathrm{OH})_{2}$ with a $5 \times 5$ supercell of graphene, and a $5 \times 5$ supercell of $\mathrm{V}_{2} \mathrm{CO}_{2}$ or $\mathrm{V}_{2} \mathrm{C}(\mathrm{OH})_{2}$ with a $6 \times 6$ supercell of graphene heterostructures were constructed according to the ground state stacking types found in our previous work. ${ }^{18}$ As depicted in Figure 1, alkali atoms are placed on the more favorable fcc sites ${ }^{37}$ with respect to the MXene structure between the heterostructure layers since the MXene-alkali atom interactions are relatively stronger.

Although it is expected that the fcc position with respect to the MXene structure is the most favorable binding site for a single intercalating alkali atom, there are 25, 16, and 9 nonequivalent sites in $\mathrm{V}_{2} \mathrm{CO}_{2}$ /graphene, $\mathrm{Ti}_{2} \mathrm{CO}_{2}$ /graphene, and $\mathrm{Sc}_{2} \mathrm{COH}_{2} /$ graphene, respectively, due to the lattice mismatch. For all these positions the binding energy per alkali atom are obtained from

$$
\begin{aligned}
E_{\mathrm{b}}= & \frac{1}{N}\left[E\left(\mathrm{M}_{2} \mathrm{CX}_{2} / \text { graphene }+ \text { alkali }\right)\right. \\
& \left.-E\left(\mathrm{M}_{2} \mathrm{CX}_{2} / \text { graphene }\right)-E(\text { alkali })\right]
\end{aligned}
$$

where $E\left(\mathrm{M}_{2} \mathrm{CX}_{2} /\right.$ graphene + alkali $)$ and $E\left(\mathrm{M}_{2} \mathrm{CX}_{2} /\right.$ graphene $)$ are the total energies of MXene/graphene heterostructures with and without alkali atoms and $E$ (alkali) is the total energy per atom of the corresponding ground state bulk structure of alkali element. $N$ is the total number of atoms in the supercell. Note that a strong binding between alkali atom and the MXene/graphene bilayer is necessary to avoid the bulk formation of alkali atoms. Figure 1 shows the binding energies for three different alkali atoms for the three considered MXene/graphene heterostructures.

In $\mathrm{Sc}_{2} \mathrm{C}(\mathrm{OH})_{2}$ /graphene the binding energies for different nonequivalent fcc sites range between -0.05 and $-0.11 \mathrm{eV} /$ atom for $\mathrm{Na},+0.81$ and $+0.71 \mathrm{eV} /$ atom for $\mathrm{K}$, and -0.05 and $-0.11 \mathrm{eV} /$ atom for $\mathrm{Ca}$. Positive binding energies for $\mathrm{K}$ implies intercalation of $\mathrm{K}$ atoms is not favorable, while very small values are found also for $\mathrm{Na}$ and $\mathrm{Ca}$ unlike for the smaller $\mathrm{Li}$ atom $(-0.94 \mathrm{eV} /$ atom $) .^{18}$ This implies that the $\mathrm{Sc}_{2} \mathrm{C}(\mathrm{OH})_{2} /$ graphene heterostructure is not promising for battery applications and is therefore discarded from further calculations.

In $\mathrm{V}_{2} \mathrm{CO}_{2}$ /graphene the binding energies range between -1.87 and $-2.05 \mathrm{eV} /$ atom for $\mathrm{Na},-1.10$ and $-1.30 \mathrm{eV} /$ atom for $\mathrm{K}$, and -3.79 and $-4.01 \mathrm{eV} /$ atom for $\mathrm{Ca}$, while in $\mathrm{Ti}_{2} \mathrm{CO}_{2} /$ graphene they range between -0.98 and $-1.11 \mathrm{eV} /$ atom for $\mathrm{Na},-0.28$ and $-0.39 \mathrm{eV} /$ atom for $\mathrm{K}$, and -2.31 and -2.51 $\mathrm{eV} /$ atom for $\mathrm{Ca}$. Accordingly, $\mathrm{V}_{2} \mathrm{CO}_{2}$ /graphene heterostructures have higher binding energies than $\mathrm{Ti}_{2} \mathrm{CO}_{2} /$ graphene and the binding energies are on the order of $\mathrm{Ca}>(\mathrm{Li})^{18}>\mathrm{Na}>\mathrm{K}$ for alkali atoms. When we compare the energies for the different nonequivalent sites in supercell, the binding energies for these sites only differ by maximum $0.2 \mathrm{eV} /$ atom.

Next, we investigated the effect of higher concentrations of alkali ions. Here, the concentration is defined as the ratio of the number of alkali atoms and the number of formula units of $\mathrm{M}_{2} \mathrm{CX}_{2}$ in the heterostructures (e.g., $100 \%$ corresponds to one alkali ion for each formula unit). In general, it is expected that, as the concentration increases, the average binding energy decreases gradually as in the case of $\mathrm{Na}$ intercalation in $\mathrm{V}_{2} \mathrm{CO}_{2}$ /graphene heterostructure (see Figure 2). This is due to the weaker electrostatic attraction between the MXene/ graphene host and the alkali cations and the stronger alkalialkali repulsion at higher concentrations due to the reduction
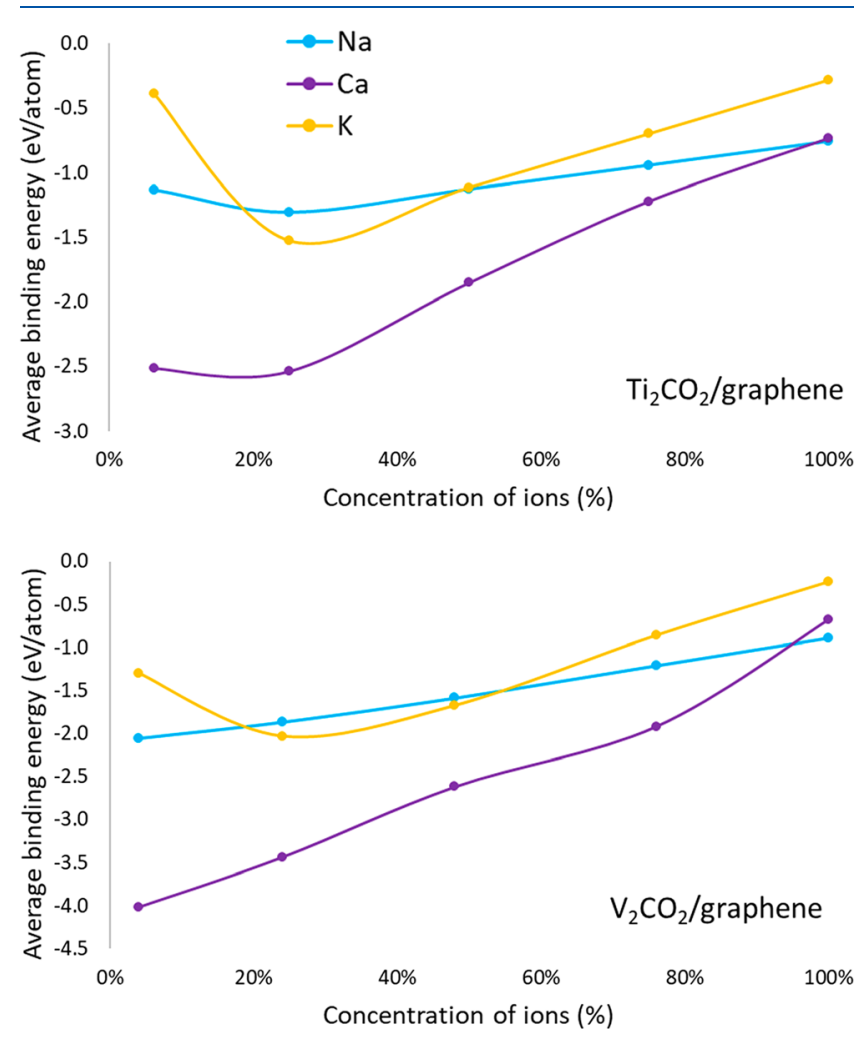

Figure 2. Average binding energy versus alkali atom concentration for $\mathrm{Ti}_{2} \mathrm{CO}_{2} /$ graphene and $\mathrm{V}_{2} \mathrm{CO}_{2} /$ graphene heterostructures. The concentration is defined as the ratio of the number of alkali atoms and the number of formula units of $\mathrm{M}_{2} \mathrm{CX}_{2}$ in the heterostructures. 
of interatomic distances between positively charged ions. The reduction of charge transfer from $\mathrm{K}$ or $\mathrm{Ca}$ atom to MXene/ graphene heterostructures at high concentrations correlates well with these interactions (see Figure 3). Notice that, for $\mathrm{Na}$
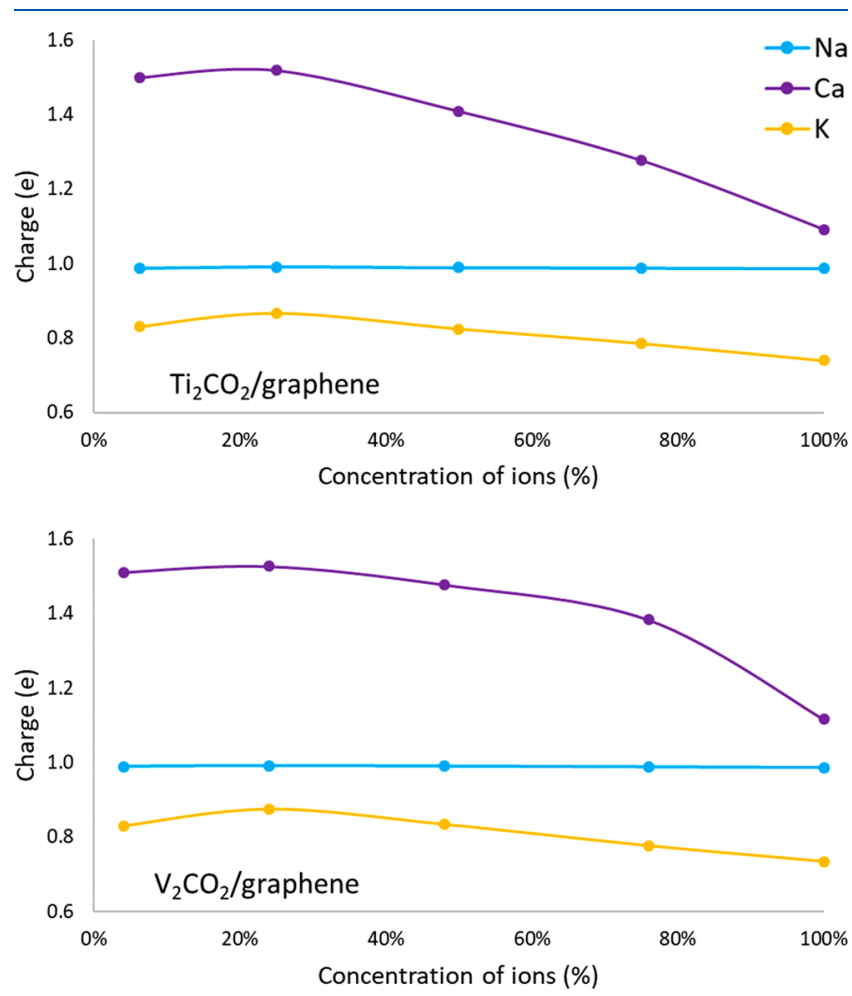

Figure 3. Graphs for the amount of charge transferred from alkali ion to MXene/graphene with respect to alkali atom concentration for $\mathrm{Ti}_{2} \mathrm{CO}_{2} /$ graphene and $\mathrm{V}_{2} \mathrm{CO}_{2} /$ graphene heterostructures. The concentration is defined as the ratio of the number of alkali atoms and the number of formula units of $\mathrm{M}_{2} \mathrm{CX}_{2}$ in the heterostructures.

atom, there is no change in charge transfer with increasing concentration and the $\mathrm{Na}-\mathrm{Na}$ repulsion effect is lower due to its smaller size. Thus, the slope of the decrease in average binding energy is smaller than the others for $\mathrm{Na}$ in both MXene/graphene heterostructures.

For $\mathrm{K}$ intercalation it can be seen from Figure 2 that average binding energy first increases at lower concentrations. This is because of the larger size of $\mathrm{K}$, it distorts the graphene sheet and the interlayer distance between Mxene and graphene layers increases more as compared to $\mathrm{Na}$ and $\mathrm{Ca}$ atoms; see Table 1. The sharpness of this effect is maximum for $\mathrm{Ti}_{2} \mathrm{CO}_{2} /$ graphene heterostructure consistent with the largest increase in interlayer spacing by $0.81 \AA$ for larger $\mathrm{K}$ atom. The change in interlayer space for $\mathrm{Ti}_{2} \mathrm{CO}_{2} /$ graphene is 2 times more than that of $\mathrm{V}_{2} \mathrm{CO}_{2}$ /graphene; however, it is probably largely due to the smaller supercell of the former due to the periodic boundary conditions, as at full coverage concentrations, interlayer distances become very similar and it is even larger by around $0.05 \AA$ for $\mathrm{V}_{2} \mathrm{CO}_{2}$ /graphene.

For comparison, we also calculated alkali atom intercalation between MXene bilayers. The single atom binding energy values are found to be higher for $\mathrm{Na}$ and $\mathrm{Ca}$ while they are smaller for $\mathrm{K}$ as compared to previous cases with heterostructures. However, for $100 \%$ concentration the binding energies are all larger; see Table 1 . The higher binding energy values are expected due to the stronger interaction
Table 1. Comparison of Different MXene Bilayer and Heterostructure Systems As Investigated in the Present Work $^{a}$

\begin{tabular}{|c|c|c|c|c|c|}
\hline \multirow[b]{2}{*}{ system } & \multicolumn{2}{|c|}{$\begin{array}{l}\text { single alkali } \\
\text { atom }\end{array}$} & \multicolumn{2}{|c|}{$\begin{array}{c}\text { full } \\
\text { concentration }\end{array}$} & \multirow[b]{2}{*}{$\begin{array}{l}\text { capacity } \\
(\mathrm{mAh} / \mathrm{g})\end{array}$} \\
\hline & $d(\AA)$ & $\begin{array}{c}E_{\mathrm{b}} \\
(\mathrm{eV})\end{array}$ & $d(\AA)$ & $\begin{array}{c}E_{\mathrm{b}} \\
(\mathrm{eV})\end{array}$ & \\
\hline \multicolumn{6}{|l|}{$\mathrm{Ti}_{2} \mathrm{CO}_{2}$} \\
\hline $\mathrm{Ti}_{2} \mathrm{CO}_{2}$ bilayer $+\mathrm{Na}$ & 2.75 & -1.72 & 3.07 & -2.14 & 164.67 \\
\hline $\begin{array}{l}\mathrm{Ti}_{2} \mathrm{CO}_{2} / \text { graphene }+ \\
\mathrm{Na}\end{array}$ & 3.27 & -1.11 & 3.75 & -0.76 & 240.07 \\
\hline $\mathrm{Ti}_{2} \mathrm{CO}_{2}$ bilayer $+\mathrm{K}$ & 3.95 & -0.37 & 3.88 & -1.56 & 149.84 \\
\hline $\mathrm{Ti}_{2} \mathrm{CO}_{2} /$ graphene $+\mathrm{K}$ & 3.88 & -0.39 & 4.51 & -0.28 & 209.80 \\
\hline $\mathrm{Ti}_{2} \mathrm{CO}_{2}$ bilayer $+\mathrm{Ca}$ & 3.07 & -3.56 & 2.97 & -2.84 & 298.04 \\
\hline $\begin{array}{l}\mathrm{Ti}_{2} \mathrm{CO}_{2} / \text { graphene }+ \\
\mathrm{Ca}\end{array}$ & 3.37 & -2.55 & 3.88 & -0.73 & 416.39 \\
\hline \multicolumn{6}{|l|}{$\mathrm{V}_{2} \mathrm{CO}_{2}$} \\
\hline $\mathrm{V}_{2} \mathrm{CO}_{2}$ bilayer $+\mathrm{Na}$ & 2.70 & -2.78 & 3.08 & -2.61 & 158.70 \\
\hline $\begin{array}{l}\mathrm{V}_{2} \mathrm{CO}_{2} / \text { graphene }+ \\
\mathrm{Na}\end{array}$ & 3.12 & -2.05 & 3.76 & -0.90 & 236.69 \\
\hline $\mathrm{V}_{2} \mathrm{CO}_{2}$ bilayer $+\mathrm{K}$ & 3.85 & -0.61 & 3.89 & -1.77 & 144.88 \\
\hline $\mathrm{V}_{2} \mathrm{CO}_{2} /$ graphene $+\mathrm{K}$ & 3.40 & -1.30 & 4.51 & -0.23 & 207.22 \\
\hline $\mathrm{V}_{2} \mathrm{CO}_{2}$ bilayer $+\mathrm{Ca}$ & 2.79 & -4.01 & 2.98 & -3.02 & 288.23 \\
\hline$\underset{\mathrm{Ca}}{\mathrm{V}_{2} \mathrm{CO}_{2} / \text { graphene }+}$ & 3.19 & -5.75 & 3.91 & -0.68 & 411.31 \\
\hline
\end{tabular}

${ }^{a} d$ is the interlayer distance between layers. $E_{\mathrm{b}}$ is the binding energy of intercalating alkali atom. Only the largest binding energy value is reported here for single alkali atom intercalation in heterostructures. Theoretical gravimetric capacities are calculated from the models corresponding to an infinite multilayer structure, where the ratio of MXene and alkali layers is 1:1 for bilayer and where the graphene/ MXene/alkali ratio is 1:1:2 for heterostructures.

between alkali metals and the MXene layers than to the graphene. However, for a single $\mathrm{K}$ atom, the binding energy is significantly lower than to the corresponding heterostructure. The reason is that for a single $\mathrm{K}$ atom, the interlayer distance between MXene layers increases by $1.5 \AA$ and thus the total energy decreases due to the loss of bilayer interaction. When we compare the theoretical gravimetric capacities, MXene/ graphene heterostructures possess larger capacity values than for MXene bilayer systems; see Table 1. The largest capacity values are found for $\mathrm{Ca}$ atoms on both heterostructures with values in excess of $400 \mathrm{mAh} / \mathrm{g}$.

Nonetheless, average binding energy values are always found negative for all considered systems, which means that $\mathrm{Na}, \mathrm{Ca}$, and $\mathrm{K}$ intercalation in these heterostructures are stable, and no phase separation into individual monolayers, or the formation of bulk alkali metals, are expected. Another important point is how the structural stability of MXene/graphene heterostructures are affected by the increase of alkali ion concentration. At $100 \%$ of $\mathrm{K}$ concentration, the interlayer spacing between MXene and graphene layers increase by almost $1.5 \AA$, which may lead to a volume expansion problem ${ }^{44}$ for rechargeable ion batteries. However, the change in interlayer spacing values are much lower for $\mathrm{Na}(\sim 0.7 \AA)$ and $\mathrm{K}(\sim 0.8 \AA)$ and comparable with $\mathrm{Li}$ atom $(0.5 \AA)^{18}$ in the same heterostructures. This amount of interlayer distance separation results in approximately around $8-12 \%$ volumetric expansion in the $z$-direction, which is comparable with the $10 \%$ of traditional graphite anodes. 45

To assess the stabilities of the considered heterostructures upon alkali metal intercalation, $a b$ initio molecular dynamics (MD) simulations within an isothermal-isobaric (NPT) 


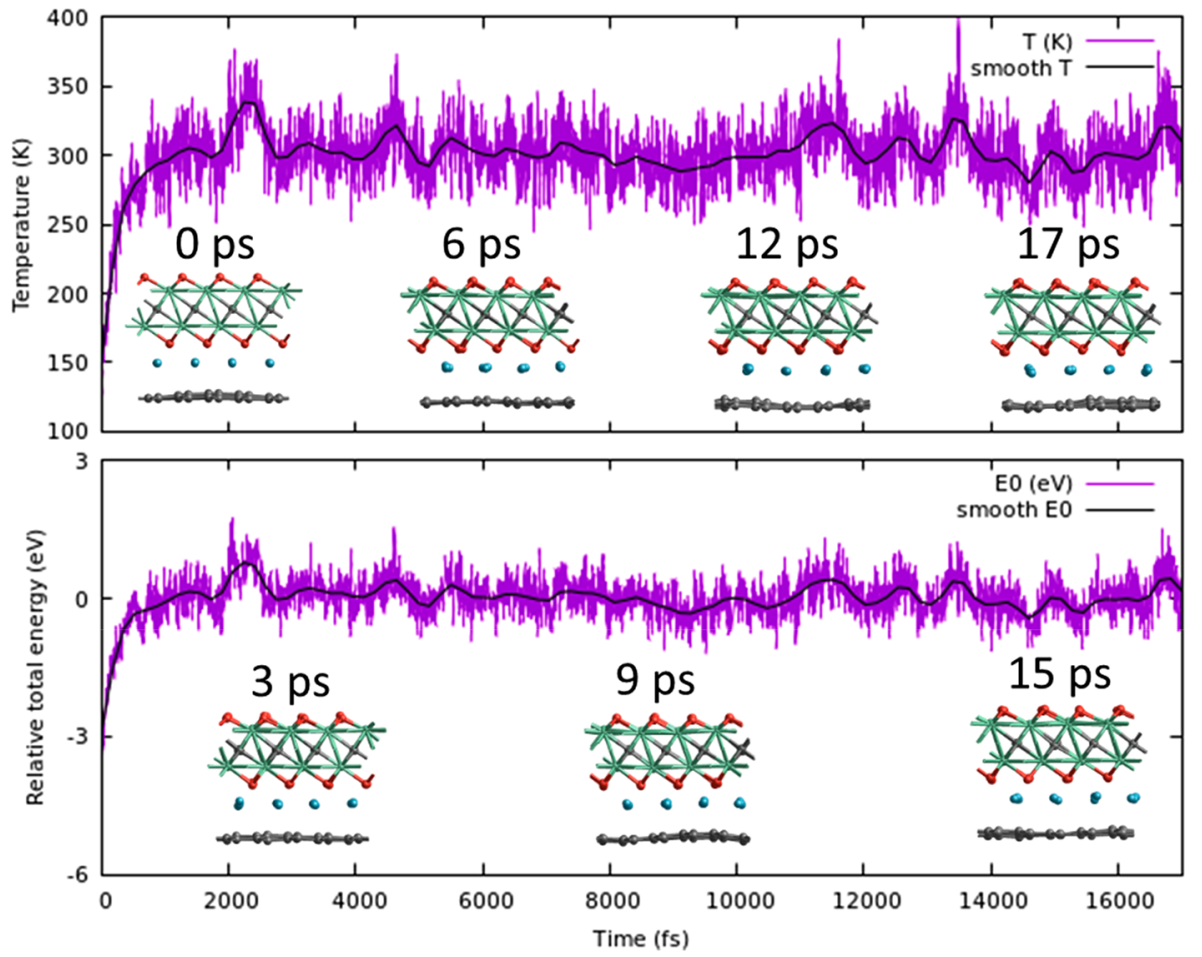

Figure 4. Temperature and total energy evaluation profiles in $\mathrm{MD}$ simulations for $\mathrm{Na}$ ion intercalation in $\mathrm{Ti}_{2} \mathrm{CO}_{2} / \mathrm{graphene}$ heterostructure. Corresponding crystal structures are given at $0,3,6,9,12,15$, and 17 ps.

ensemble were also conducted for $100 \%$ alkali atom concentration at room temperature $(300 \mathrm{~K})$ in order to see whether small perturbations will induce any structural deformation and/or alkali atom release. Figure 4 shows an example of the evolution of the total energy and temperature of $\mathrm{Na}$ intercalation in $\mathrm{Ti}_{2} \mathrm{CO}_{2}$ /graphene heterostructure system. Our simulations revealed no structural transformation for any of the considered systems during 17 ps. However, for $\mathrm{Ca}$ intercalation more significant fluctuations of the graphene sheet was observed for both heterostructures. Nevertheless, the total energy and temperature profiles shown in SI as Figure S1 suggests that MXene/graphene heterostructures are thermally stable upon intercalation.

The open-circuit-voltage value gives a measure of the performance of a battery, and it can be approximated by calculating the average voltage over a range of metal ion concentrations. The charge/discharge processes of MXene follow the common half-cell reaction vs $\mathrm{M} / \mathrm{M}^{+}$:

$$
\begin{gathered}
\left(x_{2}-x_{1}\right) \mathrm{M}^{+}+\left(x_{2}-x_{1}\right) \mathrm{e}^{-}+\left(\text {heterostructure }+x_{1} \mathrm{M}\right) \\
\left.\leftrightarrow \text { (heterostructure }+x_{1} \mathrm{M}\right)
\end{gathered}
$$

The open-circuit-voltage values are thus obtained by calculating the averaged half-cell voltage over a range of alkali metal ion $\left(M^{+}\right)$concentrations $x$ by using

$$
\begin{aligned}
& V \approx \\
& \frac{E_{\text {heterostructure }+x_{1} \mathrm{M}}-E_{\text {heterostructure }+x_{2} \mathrm{M}}+\left(x_{2}-x_{1}\right) E_{\text {bulk }(\mathrm{M})}}{\left(x_{2}-x_{1}\right) e}
\end{aligned}
$$

where $E_{\text {heterostructure }+x_{1} M}$ and $E_{\text {heterostructure }+x_{2} M}$ are the total energies of the MXene/graphene heterostructure with $x_{1}$ and $x_{2}$ alkali atom intercalated, respectively. $E_{\text {bulk(M) }}$ is the total energy of the corresponding bulk phase of the alkali atom. This simple formula estimates the voltage difference between two different alkali atom concentrations. Figure 5 shows the calculated voltage profiles of the two considered systems. Our calculations reveal that the voltage decreases gradually with increasing alkali ion concentration, except for $\mathrm{K}$ at low concentration due to its lower binding energy. The calculated average voltage corresponding to $\mathrm{K}$ ion are negative for concentrations $x$ larger than $50 \%$ for both heterostructures. Therefore, a phase transition should be expected for concentrations larger than this critical value. Similarly, for $\mathrm{Ca}$ intercalation in $\mathrm{Ti}_{2} \mathrm{CO}_{2}$ /graphene, the voltage becomes $0 \mathrm{~V}$ after $50 \%$ concentration while it drops below $0 \mathrm{~V}$ after $75 \%$ concentration. However, for $\mathrm{Na}$ in both heterostructures the voltage is found positive for all concentrations, meaning that these systems can be almost fully intercalated.

The calculated voltage values correlate well with the binding energy values. The highest voltage is found for $\mathrm{Ca}$ on $\mathrm{V}_{2} \mathrm{CO}_{2}$ / graphene as this system has the largest binding energy. Though voltage profiles are similar for $\mathrm{Na}$ and $\mathrm{Ca}$ at higher concentrations. The calculated average voltage profile is 2.47 $\mathrm{V}(1.55 \mathrm{~V})$ for $\mathrm{Ca}$ and $1.44 \mathrm{~V}(1.00 \mathrm{~V})$ for $\mathrm{Na}$ in $\mathrm{V}_{2} \mathrm{CO}_{2} /$ graphene $\left(\mathrm{Ti}_{2} \mathrm{CO}_{2} /\right.$ graphene $)$. These values are larger than the values for the same alkali ions on $\mathrm{Mo}_{2} \mathrm{C}$. ${ }^{46}$ When we compare these values with $\mathrm{Li}, \mathrm{Na}$ has smaller and $\mathrm{Ca}$ has higher average voltage profiles in the corresponding heterostructure systems. ${ }^{18}$ For $\mathrm{Ca}$ ions, approximately $50 \%$ of the capacity of $\mathrm{V}_{2} \mathrm{CO}_{2} /$ graphene $\left(\mathrm{Ti}_{2} \mathrm{CO}_{2} /\right.$ graphene $)$ is intercalated above $3 \mathrm{~V}(2 \mathrm{~V})$, with the rest intercalating at lower voltages. Our results indicate that $\mathrm{Na}$ intercalation in $\mathrm{Ti}_{2} \mathrm{CO}_{2}$ /graphene heterostructure can be exploited in low voltage applications while $\mathrm{Ca}$ intercalation of $\mathrm{V}_{2} \mathrm{CO}_{2}$ /graphene would be suitable for high charging voltage applications.

For an efficient electrode material, low diffusion barriers and thus high mobility of alkali ions are a necessity, which is a key 

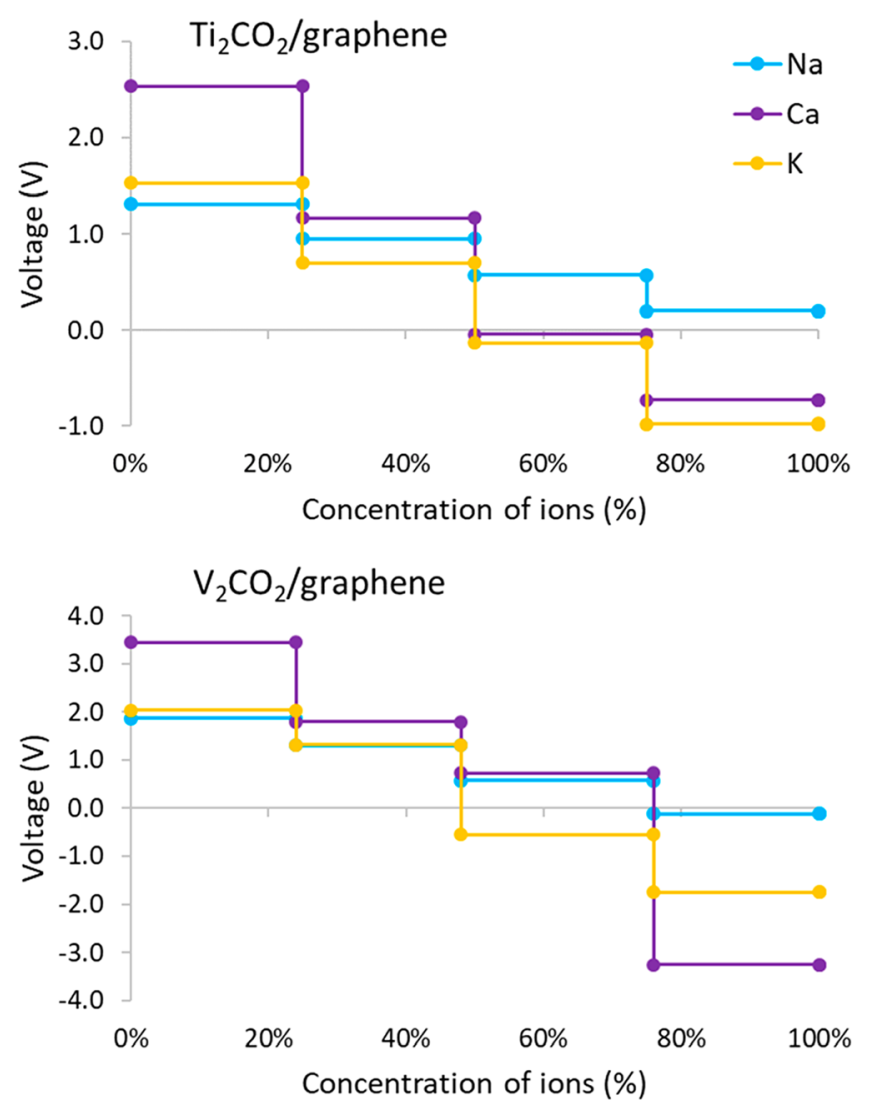

Figure 5. Voltage profiles of $\mathrm{Na}, \mathrm{Ca}$, and $\mathrm{K}$ intercalation in $\mathrm{Ti}_{2} \mathrm{CO}_{2} /$ graphene and $\mathrm{V}_{2} \mathrm{CO}_{2}$ /graphene heterostructures as a function of alkali atom concentration. The concentration is defined as the ratio of the number of alkali atoms and the number of formula units of $\mathrm{M}_{2} \mathrm{CX}_{2}$ in the heterostructures.

factor determining the rate performance during charging and discharging of a battery. Thus, we investigate the diffusion kinetics of $\mathrm{Na}, \mathrm{K}$, and $\mathrm{Ca}$ atoms in both MXene/graphene and bilayer MXene systems, by calculating the lowest migration path connecting two adjacent binding sites, with one of them being the lowest energy site. Figure 6 shows the diffusion energy barriers for the considered systems. At first glance, one can clearly see that the diffusion energy barriers are lower for MXene/graphene heterostructures than bilayer MXenes by at least a factor of 2, which indicates higher mobility and hence improved battery performance for heterostructures. In the case of heterostructures, diffusion energy profiles are less symmetric due to the nonsymmetrical matching of MXene and graphene topologies in the supercell. Even in the case of $\mathrm{Ti}_{2} \mathrm{CO}_{2} /$ graphene, local minima can be seen corresponding to metastable sites occurring due to this effect. Lower diffusion energy barriers are found for $\mathrm{Na}$ and $\mathrm{K}$ ions than $\mathrm{Ca}$ due to the much stronger binding of $\mathrm{Ca}$ intercalation as in the case of bilayer MXene systems. Too strong binding energies are expected to pin the atoms on the surface while lower binding energies effectively smooth the potential on the surface.

The diffusion energy barriers are very similar for the $\mathrm{Ti}_{2} \mathrm{CO}_{2}$ /graphene and $\mathrm{V}_{2} \mathrm{CO}_{2} /$ graphene systems. The low diffusion energy barrier for $\mathrm{K}$ and $\mathrm{Na}$ ions $(0.26$ and $0.34 \mathrm{eV}$, respectively) on both heterostructure system are comparable with, or even lower than for $\mathrm{Li}$ ion on the same heterostructure systems $(0.22-0.60 \mathrm{eV}),{ }^{18}$ on graphite $(0.5 \mathrm{eV}),{ }^{47}$ and on
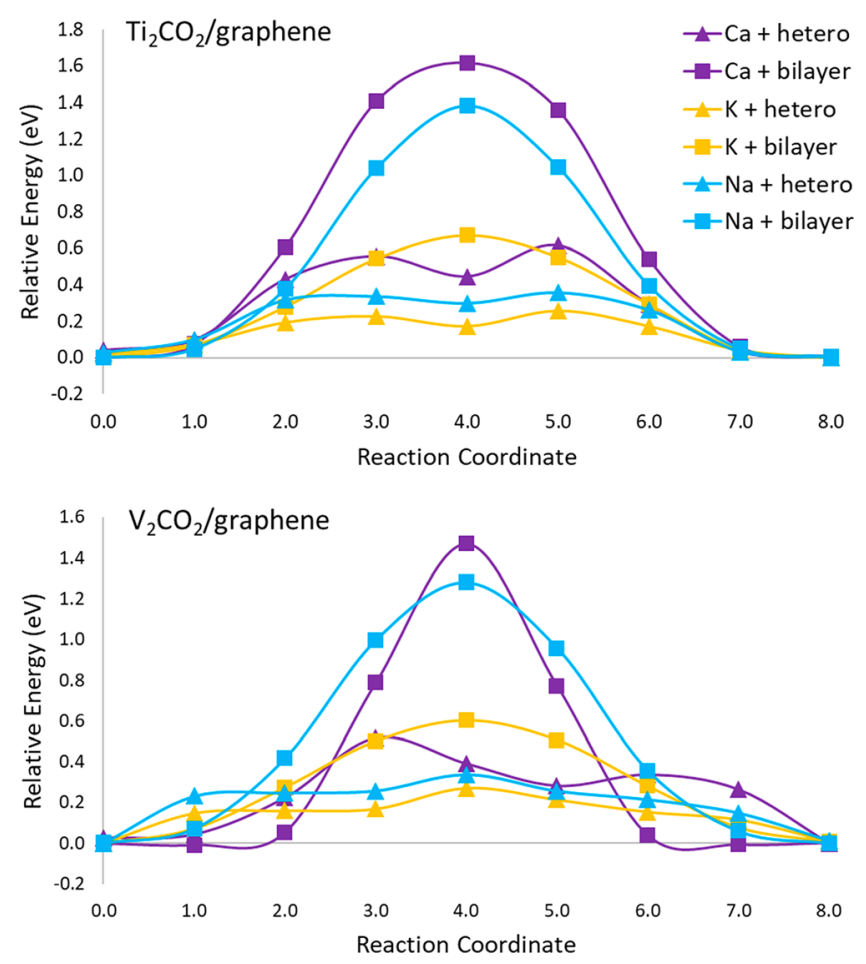

Figure 6. Calculated diffusion energy profiles for $\mathrm{Na}$, $\mathrm{Ca}$, and $\mathrm{K}$ atom for bilayer $\mathrm{Ti}_{2} \mathrm{CO}_{2}$ and $\mathrm{V}_{2} \mathrm{CO}_{2}$ and their graphene heterostructures.

commercially used anode materials based on $\mathrm{TiO}_{2}(0.35-$ $0.55),{ }^{48-50}$ even though they are heavier and larger than $\mathrm{Li}$ ion.

In summary, we systematically investigated intercalation properties of alkali metals $(\mathrm{Na}, \mathrm{K}$, and $\mathrm{Ca}$ ) in MXene/ graphene heterostructures with $\mathrm{OH}$ terminated $\mathrm{Sc}_{2} \mathrm{C}$, and $\mathrm{O}$ terminated $\mathrm{Ti}_{2} \mathrm{C}$ and $\mathrm{V}_{2} \mathrm{C}$ MXene systems for possible ion battery applications. Single atom binding energies showed that the $\mathrm{Sc}_{2} \mathrm{C}(\mathrm{OH})_{2}$ /graphene system is not favorable for battery applications. However, both $\mathrm{Ti}_{2} \mathrm{CO}_{2}$ /graphene and $\mathrm{V}_{2} \mathrm{CO}_{2} /$ graphene systems showed favorable intercalation properties for all the considered alkali atoms, with $\mathrm{Ca}$ having the strongest binding energies while $\mathrm{K}$ has the smallest. As expected, increasing the alkali ion concentration showed a decreasing trend in average binding energies, but intercalation remained still favorable until $100 \%$ ion concentration. $A b$ initio $\mathrm{MD}$ simulations confirmed thermal stabilities of these systems at $100 \%$ ion concentration. Thus, our study confirms that the high theoretical gravimetric capacity values computed for MXene/graphene heterostructures are achievable.

Charge transfer calculations found that intercalated alkali atoms become positively charged by giving an electron to the MXene/graphene host, which is necessary for battery applications. While the charge transfer decreases for $\mathrm{Ca}$ and $\mathrm{K}$ atoms as the concentration increases, for $\mathrm{Na}$ atom almost $1 \mathrm{e}$ is transferred for all concentrations. The calculated average voltage values become negative after $50 \% \mathrm{~K}$ ion concentration, while they remain positive for $\mathrm{Na}$ until 100\%. Diffusion energy barriers confirm the advantage of heterostructures over MXene bilayer systems by decreasing the barriers with more than a factor of 2. Low diffusion energy barriers are found for $\mathrm{Na}$ and $\mathrm{K}$ systems, which implies their high charge/discharge rate in battery applications. Although the barriers are lowest for $\mathrm{K}$ ions, the interlayer spacing is increased significantly due to the larger size of the $\mathrm{K}$ atom. This may lead to volumetric expansion problems in battery applications. 
Altogether, these results lead us to conclude that the lowcost $\mathrm{Na}$ element is very promising for MXene/graphene battery applications. We found that $\mathrm{Ti}_{2} \mathrm{CO}_{2} /$ graphene would be more suitable for low voltage applications, while $\mathrm{V}_{2} \mathrm{CO}_{2}$ / graphene is advantage for higher charging voltage applications.

\section{ASSOCIATED CONTENT}

\section{S Supporting Information}

The Supporting Information is available free of charge on the ACS Publications website at DOI: 10.1021/acs.jpclett.8b03056.

Schematic figure of considered systems, ab initio molecular dynamics results (temperature and energy curves) (PDF)

\section{AUTHOR INFORMATION}

\section{Corresponding Author}

*E-mail: csevik@anadolu.edu.tr.

\section{ORCID}

Ilker Demiroglu: 0000-0001-7801-4566

Deniz Çak1r: 0000-0003-3315-5204

Cem Sevik: 0000-0002-2412-9672

\section{Notes}

The authors declare no competing financial interest.

\section{ACKNOWLEDGMENTS}

We acknowledge the support from the TUBITAK (116F080) and the BAGEP Award of the Science Academy. Part of this work was supported by the FLAG-ERA project TRANS-2DTMD. A part of this work was supported by University of North Dakota Early Career Award (Grant number: 206224000-02624). We also acknowledge financial support from ND EPSCoR through NSF grant OIA-1355466. Computational resources were provided by the High Performance and Grid Computing Center (TRGrid e-Infrastructure) of TUBITAK ULAKBIM, the National Center for High Performance Computing (UHeM) of Istanbul Technical University, and Computational Research Center (HPC Linux cluster) at the University of North Dakota. This work was performed, in part, at the Center for Nanoscale Materials, a U.S. Department of Energy Office of Science User Facility, and supported by the U.S. Department of Energy, Office of Science, under contract no. DE-AC02- 06CH11357.

\section{REFERENCES}

(1) Yabuuchi, N.; Kubota, K.; Dahbi, M.; Komaba, S. Research Development on Sodium-Ion Batteries. Chem. Rev. 2014, 114, 11636-11682.

(2) Pan, H.; Hu, Y. S.; Chen, L. Room-Temperature Stationary Sodium-Ion Batteries for Large-Scale Electric Energy Storage. Energy Environ. Sci. 2013, 6, 2338-2360.

(3) Palomares, V.; Serras, P.; Villaluenga, I.; Hueso, K. B.; CarreteroGonzález, J.; Rojo, T. Na-Ion Batteries, Recent Advances and Present Challenges to Become Low Cost Energy Storage Systems. Energy Environ. Sci. 2012, 5 (3), 5884.

(4) Luo, W.; Shen, F.; Bommier, C.; Zhu, H.; Ji, X.; Hu, L. Na-Ion Battery Anodes: Materials and Electrochemistry. Acc. Chem. Res. 2016, 49 (2), 231-240.

(5) Yoo, E. J.; Kim, J.; Hosono, E.; Zhou, H. S.; Kudo, T.; Honma, I. Large Reversible Li Storage of Graphene Nanosheet Families for Use in Rechargeable Lithium Ion Batteries. Nano Lett. 2008, 8 (8), 22772282.
(6) Yang, E.; Ji, H.; Jung, Y. Two-Dimensional Transition Metal Dichalcogenide Monolayers as Promising Sodium Ion Battery Anodes. J. Phys. Chem. C 2015, 119 (47), 26374-26380.

(7) Jing, Y.; Zhou, Z.; Cabrera, C. R.; Chen, Z. Metallic VS2Monolayer: A Promising 2D Anode Material for Lithium Ion Batteries. J. Phys. Chem. C 2013, 117 (48), 25409-25413.

(8) Sun, J.; Lee, H. W.; Pasta, M.; Yuan, H.; Zheng, G.; Sun, Y.; Li, Y.; Cui, Y. A Phosphorene-Graphene Hybrid Material as a HighCapacity Anode for Sodium-Ion Batteries. Nat. Nanotechnol. 2015, 10 (11), 980-985.

(9) Kulish, V. V.; Malyi, O. I.; Persson, C.; Wu, P. Phosphorene as an Anode Material for Na-Ion Batteries: A First-Principles Study. Phys. Chem. Chem. Phys. 2015, 17 (21), 13921-13928.

(10) Pan, H. Electronic Properties and Lithium Storage Capacities of Two-Dimensional Transition-Metal Nitride Monolayers. J. Mater. Chem. A 2015, 3 (43), 21486-21493.

(11) Wang, D.; Gao, Y.; Liu, Y.; Jin, D.; Gogotsi, Y.; Meng, X.; Du, F.; Chen, G.; Wei, Y. First-Principles Calculations of Ti $2 \mathrm{~N}$ and Ti 2 NT $2(\mathrm{~T}=\mathrm{O}, \mathrm{F}, \mathrm{OH})$ Monolayers as Potential Anode Materials for Lithium-Ion Batteries and Beyond. J. Phys. Chem. C 2017, 121 (24), 13025-13034.

(12) Wang, D.; Gao, Y.; Liu, Y.; Gogotsi, Y.; Meng, X.; Chen, G.; Wei, Y. Investigation of Chloride Ion Adsorption onto Ti2C MXene Monolayers by First-Principles Calculations. J. Mater. Chem. A 2017, 5 (47), 24720-24727.

(13) Xie, Y.; Dall'Agnese, Y.; Naguib, M.; Gogotsi, Y.; Barsoum, M. W.; Zhuang, H. L.; Kent, P. R. C. Prediction and Characterization of Mxene Nanosheet Anodes for Non-Lithium-Ion Batteries. ACS Nano 2014, 8 (9), 9606-9615.

(14) Naguib, M.; Halim, J.; Lu, J.; Cook, K. M.; Hultman, L.; Gogotsi, Y.; Barsoum, M. W. New Two-Dimensional Niobium and Vanadium Carbides as Promising Materials for Li-Ion Batteries. J. Am. Chem. Soc. 2013, 135 (43), 15966-15969.

(15) Hu, J.; Xu, B.; Yang, S. A.; Guan, S.; Ouyang, C.; Yao, Y. 2D Electrides as Promising Anode Materials for Na-Ion Batteries from First-Principles Study. ACS Appl. Mater. Interfaces 2015, 7 (43), 24016-24022.

(16) Ashton, M.; Hennig, R. G.; Sinnott, S. B. Computational Characterization of Lightweight Multilayer MXene Li-Ion Battery Anodes. Appl. Phys. Lett. 2016, 108 (2), 023901.

(17) Ashton, M.; Mathew, K.; Hennig, R. G.; Sinnott, S. B. Predicted Surface Composition and Thermodynamic Stability of MXenes in Solution. J. Phys. Chem. C 2016, 120 (6), 3550-3556.

(18) Aierken, Y.; Sevik, C.; Gülseren, O.; Peeters, F. M.; Çakir, D. MXenes/Graphene Heterostructures for Li Battery Applications: A First Principles Study. J. Mater. Chem. A 2018, 6 (5), 2337-2345.

(19) Pomerantseva, E.; Gogotsi, Y. Two-Dimensional Heterostructures for Energy Storage. Nat. Energy 2017, 2 (7), 17089.

(20) Wang, X.; Weng, Q.; Yang, Y.; Bando, Y.; Golberg, D. Hybrid Two-Dimensional Materials in Rechargeable Battery Applications and Their Microscopic Mechanisms. Chem. Soc. Rev. 2016, 45, 40424073.

(21) Ren, C. E.; Zhao, M. Q.; Makaryan, T.; Halim, J.; Boota, M.; Kota, S.; Anasori, B.; Barsoum, M. W.; Gogotsi, Y. Porous TwoDimensional Transition Metal Carbide (MXene) Flakes for HighPerformance Li-Ion Storage. ChemElectroChem 2016, 3 (5), 689-693.

(22) Aïssa, B.; Ali, A.; Mahmoud, K. A.; Haddad, T.; Nedil, M. Transport Properties of a Highly Conductive 2D Ti $3 \mathrm{C} 2 \mathrm{~T} \mathrm{x}$ MXene/Graphene Composite. Appl. Phys. Lett. 2016, 109 (4), 043109.

(23) Ma, Z.; Zhou, X.; Deng, W.; Lei, D.; Liu, Z. 3D Porous MXene (Ti3C2)/Reduced Graphene Oxide Hybrid Films for Advanced Lithium Storage. ACS Appl. Mater. Interfaces 2018, 10 (4), 36343643.

(24) Couly, C.; Alhabeb, M.; Van Aken, K. L.; Kurra, N.; Gomes, L.; Navarro-Suárez, A. M.; Anasori, B.; Alshareef, H. N.; Gogotsi, Y. Asymmetric Flexible MXene-Reduced Graphene Oxide Micro-Supercapacitor. Adv. Electron. Mater. 2018, 4 (1), 1700339. 
(25) Yue, Y.; Liu, N.; Ma, Y.; Wang, S.; Liu, W.; Luo, C.; Zhang, H.; Cheng, F.; Rao, J.; Hu, X.; et al. Highly Self-Healable 3D Microsupercapacitor with MXene-Graphene Composite Aerogel. ACS Nano 2018, 12 (5), 4224-4232.

(26) Wu, X.; Wang, Z.; Yu, M.; Xiu, L.; Qiu, J. Stabilizing the MXenes by Carbon Nanoplating for Developing Hierarchical Nanohybrids with Efficient Lithium Storage and Hydrogen Evolution Capability. Adv. Mater. 2017, 29 (24), 1607017.

(27) Yan, J.; Ren, C. E.; Maleski, K.; Hatter, C. B.; Anasori, B.; Urbankowski, P.; Sarycheva, A.; Gogotsi, Y. Flexible MXene/ Graphene Films for Ultrafast Supercapacitors with Outstanding Volumetric Capacitance. Adv. Funct. Mater. 2017, 27 (30), 1701264.

(28) Yang, Q.; Xu, Z.; Fang, B.; Huang, T.; Cai, S.; Chen, H.; Liu, Y.; Gopalsamy, K.; Gao, W.; Gao, C. MXene/Graphene Hybrid Fibers for High Performance Flexible Supercapacitors. J. Mater. Chem. A 2017, 5 (42), 22113-22119.

(29) Du, Y.-T.; Kan, X.; Yang, F.; Gan, L.; Schwingenschlögl, U. MXene/Graphene Heterostructures as High-Performance Electrodes for Li Ion Batteries. ACS Appl. Mater. Interfaces 2018, 10, 32867.

(30) Paul, P.; Chakraborty, P.; Das, T.; Nafday, D.; Saha-Dasgupta, T. Properties at the Interface of Graphene and Ti2 C MXene. Phys. Rev. B: Condens. Matter Mater. Phys. 2017, 96 (3), 035435.

(31) Kresse, G.; Hafner, J. Ab Initio Molecular Dynamics for Liquid Metals. Phys. Rev. B: Condens. Matter Mater. Phys. 1993, 47 (1), 558561.

(32) Kresse, G.; Hafner, J. Ab Initio Molecular-Dynamics Simulation of the Liquid-Metalamorphous- Semiconductor Transition in Germanium. Phys. Rev. B: Condens. Matter Mater. Phys. 1994, 49 (20), 14251-14269.

(33) Perdew, J. P.; Burke, K.; Ernzerhof, M. Generalized Gradient Approximation Made Simple. Phys. Rev. Lett. 1996, 77 (18), 38653868.

(34) Kresse, G.; Joubert, D. From Ultrasoft Pseudopotentials to the Projector Augmented-Wave Method. Phys. Rev. B: Condens. Matter Mater. Phys. 1999, 59 (3), 1758-1775.

(35) Blöchl, P. E. Projector Augmented-Wave Method. Phys. Rev. B: Condens. Matter Mater. Phys. 1994, 50 (24), 17953-17979.

(36) Grimme, S.; Antony, J.; Ehrlich, S.; Krieg, H. A Consistent and Accurate Ab Initio Parametrization of Density Functional Dispersion Correction (DFT-D) for the 94 Elements H-Pu. J. Chem. Phys. 2010, 132 (15), 154104.

(37) Grimme, S.; Ehrlich, S.; Goerigk, L. Effect of the Damping Function in Dispersion Corrected Density Functional Theory. J. Comput. Chem. 2011, 32 (7), 1456-1465.

(38) Henkelman, G.; Uberuaga, B. P.; Jónsson, H. Climbing Image Nudged Elastic Band Method for Finding Saddle Points and Minimum Energy Paths. J. Chem. Phys. 2000, 113 (22), 9901-9904.

(39) Henkelman, G.; Jónsson, H. Improved Tangent Estimate in the Nudged Elastic Band Method for Finding Minimum Energy Paths and Saddle Points. J. Chem. Phys. 2000, 113 (22), 9978-9985.

(40) Hernández, E. Metric-Tensor Flexible-Cell Algorithm for Isothermal-Isobaric Molecular Dynamics Simulations. J. Chem. Phys. 2001, 115 (22), 10282-10290.

(41) Computer Simulation of Liquids, Second ed.; Allen, Michael P., Tildesley, Dominic J., Eds.; Oxford University Press, 2017.

(42) Parrinello, M.; Rahman, A. Polymorphic Transitions in Single Crystals: A New Molecular Dynamics Method. J. Appl. Phys. 1981, 52 (12), 7182-7190.

(43) Parrinello, M.; Rahman, A. Crystal Structure and Pair Potentials: A Molecular-Dynamics Study. Phys. Rev. Lett. 1980, 45 (14), 1196-1199.

(44) Sun, Y.; Liu, N.; Cui, Y. Promises and Challenges of Nanomaterials for Lithium-Based Rechargeable Batteries. Nat. Energy 2016, 1, 16071.

(45) Ohzuku, T. Formation of Lithium-Graphite Intercalation Compounds in Nonaqueous Electrolytes and Their Application as a Negative Electrode for a Lithium Ion (Shuttlecock) Cell. J. Electrochem. Soc. 1993, 140 (9), 2490.
(46) Çakir, D.; Sevik, C.; Gülseren, O.; Peeters, F. M. Mo2C as a High Capacity Anode Material: A First-Principles Study. J. Mater. Chem. A 2016, 4 (16), 6029-6035.

(47) Thinius, S.; Islam, M. M.; Heitjans, P.; Bredow, T. Theoretical Study of Li Migration in Lithium-Graphite Intercalation Compounds with Dispersion-Corrected DFT Methods. J. Phys. Chem. C 2014, 118 (5), 2273-2280.

(48) Lunell, S.; Stashans, A.; Ojamäe, L.; Lindström, H.; Hagfeldt, A. $\mathrm{Li}$ and $\mathrm{Na}$ Diffusion in $\mathrm{TiO} 2$ from Quantum Chemical Theory versus Electrochemical Experiment. J. Am. Chem. Soc. 1997, 119 (31), 7374-7380.

(49) Lindström, H.; Södergren, S.; Solbrand, A.; Rensmo, H.; Hjelm, J.; Hagfeldt, A.; Lindquist, S.; Lindstro, H.; So, S.; Solbrand, A.; et al. $\mathrm{Li}+$ Ion Insertion in $\mathrm{TiO}_{2}$ (Anatase). 1. Chronoamperometry on CVD Films and Nanoporous Films. J. Phys. Chem. B 1997, 101 (39), $7710-7716$

(50) Olson, C. L.; Nelson, J.; Saiful Islam, M. Defect Chemistry, Surface Structures, and Lithium Insertion in Anatase $\mathrm{TiO}_{2}$. J. Phys. Chem. B 2006, 110 (20), 9995-10001. 